\title{
A New Information Priority Accumulated Grey Model with Hyperbolic Sinusoidal Term and its Application
}

\author{
Xue Tian ${ }^{1}$ Wenqing $\mathrm{Wu}^{1,2,{ }^{*}} \mid$ Xin $\mathrm{Ma}^{1} \mid$ Peng Zhang ${ }^{1}$ \\ ${ }^{1}$ School of Science, Southwest University of Science and Technology, 621010, Mianyang, China \\ ${ }^{2}$ Visual Computing and Virtual Reality Key Laboratory of Sichuan Province, Sichuan Normal University, 610068, Chengdu, China \\ *Corresponding author: wwqing0704@163.com
}

Received 21 July 2021; Revised 9 September 2021; Accepted 10 September 2021

\begin{abstract}
Compared to fossil fuels, natural gas is cleaner energy, which has developed rapidly in recent years. Studying the urban supply of natural gas has implications for the development of natural gas. In this paper, the new information priority accumulation method is integrated into the grey forecasting model with the hyperbolic sinusoidal driving term, and then the new grey model is used to predict the urban natural gas supply. The system's linear parameters are calculated by the least square estimation method, and the optimal parameter of the new information accumulated priority is determined by the Whale Optimization Algorithm. Finally, the supply of urban gas is forecasted using the proposed model, and comparative analyses with the four other forecasting models are presented.
\end{abstract}

Keywords: Natural gas; grey forecasting; new information priority accumulation; hyperbolic sinusoidal; optimization algorithm

\section{Introduction}

Natural gas has become one of the most prevalent forms of energy. Natural gas is widely used in many ways, such as generating electricity, the chemical industry, etc. Because natural gas produces less pollution, is convenient to deliver, and is more affordable than other gases, it is widely applied in our daily lives. Nowadays, natural gas is being delivered to the countryside or distant mountains villages, increasing its consumption year by year. But is the gas supply enough to meet the needs of the urban population? Thus, studying the supply of urban natural gas is of significant importance.

In the 1980s, Deng (1982) proposed the Grey System Theory and opened the door to a new discipline. From then on, the theory has seen application in various fields, especially the energy sector (Wu \& Ma, 2019a; 2019b; 2018). Grey forecasting theory is an important branch of it, as it just needs more than four data to make a prediction and obtain acceptable accuracy. In recent years, scholars have proposed a number of models with different structures based on the typical grey prediction model GM $(1,1)$. According to the structure of the GM $(1,1)$ grey model, Cui et al. (2013) and Xie et al. (2013) introduced a new grey action $b t$ replacing the original grey action $b$ into the grey model to propose continuous grey model NGM $(1,1, k)$ and discrete grey model NDGM 
$(1,1, k)$. Based on their work, Chen $e t$ al.(2014) introduced the $b t+c$ term into the grey model to construct a non-homogeneous grey prediction model called NGM $(1,1, k, c)$. Recently, Zhang et al. (2019) proposed power-driven and incomplete Gamma grey action term into the grey model.. Besides the univariate form, many scholars also research the multivariate grey models, such as the GM (1,N) model (Deng, 2012; Tien, 2005). Ma et al. (2014) introduced the nonlinear term into the GM $(1, \mathrm{~N})$ model to propose the nonlinear grey Bernoulli GM $(1, \mathrm{~N})$ model.

In traditional grey models, the integer order accumulation method is used to the original sequences to obtain a new increasing sequence which is called the first accumulated generating operation (1-AGO). Followed by the idea of the 1-AGO, there are three other ways to deal with original data sequences. Xia et al. (2020) combined the new information priority accumulation into the grey model to predict short-term wind turbine capacity. The fractional-order accumulation is introduced into the grey model in 2013 by Wu et al. (2013). Ma et al. (2019) proposed a new fractional accumulation definition and introduced it into the traditional grey model GM $(1,1)$ to construct the CFGM $(1,1)$.

The detailed algorithm of new information priority accumulation was proposed by Zhou et al. (2017). Wu et al. (2018) demonstrated that in the new information priority accumulation, the weight of new information should be larger than older information. They pointed out that giving new information more weight would increase the grey model's accuracy. Inspired by the abovementioned grey models and the advantage of the new information priority accumulation, we propose a new information priority accumulation-based grey forecasting model, abbreviated as NISinHGM $(1,1)$. The current study aims to combine the new information priority accumulation into a grey model with the hyperbolic sinusoidal driving term and then forecast the total urban natural gas supply. The contributions of our paper are summarized as follows. Firstly, we introduce and recall the new information priority accumulation method. Secondly, we use the Whale Optimization Algorithm (WOA) to calculate the parameter, and then a new model, NISinHGM $(1,1)$, is built. The properties of the new model are systematically analyzed. Last but not least, the proposed grey model is applied to the natural gas of urban supply, and the results are compared with the four other grey prediction models.

\section{New information priority accumulation method}

Definition 1. Let the negative sequence be $X^{(0)}=\left(x^{(0)}(1), x^{(0)}(2), \ldots, x^{(0)}(n)\right)$, the first-order priority accumulation sequence of new information is expressed as

$$
x^{(1)}(k)=\sum_{i=1}^{k} \lambda^{k-i} x^{(0)}(i), \lambda \in[0,1],
$$

so the first order generation sequence is $X^{(1)}=\left(x^{(1)}(1), x^{(1)}(2), \ldots, x^{(1)}(n)\right)$, which writes in a matrix form as follows

$$
\left(\begin{array}{c}
x^{(1)}(1) \\
x^{(1)}(2) \\
x^{(1)}(3) \\
\vdots \\
x^{(1)}(n-2) \\
x^{(1)}(n-1) \\
x^{(1)}(n)
\end{array}\right)=\left(\begin{array}{ccccccc}
1 & 0 & 0 & \cdots & 0 & 0 & 0 \\
\lambda & 1 & 0 & \cdots & 0 & 0 & 0 \\
\lambda^{2} & \lambda & 1 & \cdots & 0 & 0 & 0 \\
\vdots & \vdots & \vdots & \ddots & \vdots & \vdots & \vdots \\
\lambda^{n-3} & \lambda^{n-4} & \lambda^{n-5} & \cdots & 1 & 0 & 0 \\
\lambda^{n-2} & \lambda^{n-3} & \lambda^{n-4} & \cdots & \lambda & 1 & 0 \\
\lambda^{n-1} & \lambda^{n-2} & \lambda^{n-3} & \cdots & \lambda^{2} & \lambda & 1
\end{array}\right)\left(\begin{array}{c}
x^{(0)}(1) \\
x^{(0)}(2) \\
x^{(0)}(3) \\
\vdots \\
x^{(0)}(n-2) \\
x^{(0)}(n-1) \\
x^{(0)}(n)
\end{array}\right) .
$$


Definition 2. Inverse operation is performed on first-order new information accumulation obtain the values

$$
x^{(0)}(k)=x^{(1)}(k)-\lambda x^{(1)}(k-1),
$$

which can also be written in matrix form as

$$
\left(\begin{array}{c}
x^{(0)}(1) \\
x^{(0)}(2) \\
x^{(0)}(3) \\
\vdots \\
x^{(0)}(n-2) \\
x^{(0)}(n-1) \\
x^{(0)}(n)
\end{array}\right)=\left(\begin{array}{ccccccc}
1 & 0 & 0 & \cdots & 0 & 0 & 0 \\
-\lambda & 1 & 0 & \cdots & 0 & 0 & 0 \\
0 & -\lambda & 1 & \cdots & 0 & 0 & 0 \\
\vdots & \vdots & \vdots & \ddots & \vdots & \vdots & \vdots \\
0 & 0 & 0 & \cdots & 1 & 0 & 0 \\
0 & 0 & 0 & \cdots & -\lambda & 1 & 0 \\
0 & 0 & 0 & \cdots & 0 & -\lambda & 1
\end{array}\right)\left(\begin{array}{c}
x^{(1)}(1) \\
x^{(1)}(2) \\
x^{(1)}(3) \\
\vdots \\
x^{(1)}(n-2) \\
x^{(1)}(n-1) \\
x^{(1)}(n)
\end{array}\right) .
$$

The weight of the first-order new information sequence can be adjusted through the parameter $\lambda$, making the weight of the new information larger than the old information.

\section{The NISinHGM $(1,1)$ model}

Definition 3. Based on first-order new information accumulation data sequence, the classical grey prediction model, the ordinary differential equation theory, and the hyperbolic sine function, a new grey prediction model NISinHGM $(1,1)$ is proposed. The whitening equation of the new model is given by

$$
\frac{d x^{(1)}(t)}{d t}+a x^{(1)}(t)=b \sinh t+c,
$$

where $\sinh t=\frac{e^{t}-e^{-t}}{2}$

Theorem 1. The time response function of the grey model NISinHGM $(1,1)$ is

$$
\begin{aligned}
\hat{x}^{(1)}(k)= & \frac{b}{2(a+1)}\left(e^{k}-e^{-a(k-1)+1}\right)-\frac{b}{2(a-1)}\left(e^{-k}-e^{-a(k-1)-1}\right) \\
& +\left(x^{(0)}(1)-\frac{c}{a}\right) e^{-a(k-1)}+\frac{c}{a},(k=1,2, \ldots),
\end{aligned}
$$

and the restored value of $\hat{x}^{(0)}(k)$ is

$$
\begin{aligned}
\hat{x}^{(0)}(k)= & \frac{b e^{k-1}}{2(a+1)}(e-\lambda)-\frac{b e^{-k+1}}{2(a-1)}\left(e^{-1}-\lambda\right)+\frac{c}{a}(1-\lambda) \\
& +\left(e^{-a}-\lambda\right) e^{-a(k-2)}\left(-\frac{b e}{2(a+1)}+\frac{b e^{-1}}{2(a-1)}+x^{(0)}(1)-\frac{c}{a}\right)\left(e^{-a}-\lambda\right) e^{-a(k-2)}, \\
& (k=1,2, \ldots),
\end{aligned}
$$


with $\lambda \in[0,1]$.

Proof 1. Transforming the homogeneous equation of Eq. (3) into

$$
\frac{d x^{(1)}(t)}{d t}=-a x^{(1)}(t)
$$

By using the constant variation method, we calculate the first-order differential equation

$$
x^{(1)}(t)=u(t) e^{-a t} .
$$

Substituting the expression of $x^{(1)}(t)$ into the original Eq. (3), one obtains

$$
u^{\prime}(t)=(b \sinh t+c) \times e^{a t} .
$$

Integrating $u^{\prime}(t)$ on the left and the right sides, one can obtain

$$
u(t)=\frac{b}{2(a+1)}\left[e^{(a+1) t}-e^{a+1}\right]-\frac{b}{2(a-1)}\left[e^{(a-1) t}-e^{a-1}\right]+\frac{c}{a}\left(e^{a t}-e^{a}\right)+x^{(1)}(1) e^{a} .
$$

Substituting $u(t)$ into Eq. (7) will produce

$$
\begin{aligned}
x^{(1)}(t)=u(t) e^{-a t} & =\frac{b}{2(a+1)}\left[e^{t}-e^{-a(t-1)+1}\right]-\frac{b}{2(a-1)} \\
& \times\left[e^{-t}-e^{-a(t-1)-1}\right]+\left(x^{(0)}(1)-\frac{c}{a}\right) e^{-a(t-1)}+\frac{c}{a} .
\end{aligned}
$$

Let $t=k$ and one can directly calculate the time response function

$$
\begin{aligned}
\hat{x}^{(1)}(k)= & \frac{b}{2(a+1)}\left(e^{k}-e^{-a(k-1)+1}\right)-\frac{b}{2(a-1)}\left(e^{-k}-e^{-a(k-1)-1}\right) \\
& +\left(x^{(0)}(1)-\frac{c}{a}\right) e^{-a(k-1)}+\frac{c}{a},(k=1,2, \ldots),
\end{aligned}
$$

Moreover, the restored values of $\hat{x}^{(0)}(k)$ can be obtained with the expression of $\hat{x}^{(1)}(k)$ and the inverse new information priority accumulation

$$
\begin{aligned}
\hat{x}^{(0)}(k)= & \frac{b e^{k-1}}{2(a+1)}(e-\lambda)-\frac{b e^{-k+1}}{2(a-1)}\left(e^{-1}-\lambda\right)+\frac{c}{a}(1-\lambda) \\
& +\left(e^{-a}-\lambda\right) e^{-a(k-2)}\left(-\frac{b e}{2(a+1)}+\frac{b e^{-1}}{2(a-1)}+x^{(0)}(1)-\frac{c}{a}\right)\left(e^{-a}-\lambda\right) e^{-a(k-2)}, \\
& (k=1,2, \ldots),
\end{aligned}
$$

Theorem 2. The system's linear parameters in the newly proposed grey prediction model NISinHGM $(1,1)$ can be expressed as

$$
(a, b, c)^{T}=\left(B^{T} B\right)^{-1}\left(B^{T} Y\right)
$$


where

$$
B=\left(\begin{array}{ccc}
-z^{(1)}(2) & \cosh 2-\cosh 1 & 1 \\
-z^{(1)}(3) & \cosh 3-\cosh 2 & 1 \\
\vdots & \vdots & \vdots \\
-z^{(1)}(r) & \cosh r-\cosh (r-1) & 1
\end{array}\right), Y=\left(\begin{array}{c}
x^{(1)}(2)-x^{(1)}(1) \\
x^{(1)}(3)-x^{(1)}(2) \\
\vdots \\
x^{(1)}(r)-x^{(1)}(r-1)
\end{array}\right)
$$

Proof 2. Integrating the whitening equation (3) and organizing it, we obtain

$$
\int_{k-1}^{k} d x^{(1)}(t)+a \int_{k-1}^{k} x^{(1)}(t) d t=b \int_{k-1}^{k} \sinh t d t+\int_{k-1}^{k} c d t
$$

By applying the trapezoid formula $\int_{k-1}^{k} x^{(1)}(t) d t=\frac{x^{(1)}(k)+x^{(1)}(k-1)}{2}=z^{(1)}(k)$, one gets

$$
x^{(1)}(k)-x^{(0)}(k)=-a z^{(1)}(k)+b[\cosh k-\cosh (k-1)]+c(k=2,3, \ldots r),
$$

Writing in matrix form, it becomes

$$
\left(\begin{array}{c}
x^{(1)}(2)-x^{(1)}(1) \\
x^{(1)}(3)-x^{(1)}(2) \\
\vdots \\
x^{(1)}(r)-x^{(1)}(r-1)
\end{array}\right)=\left(\begin{array}{ccc}
-z^{(1)}(2) & \cosh 2-\cosh 1 & 1 \\
-z^{(1)}(3) & \cosh 3-\cosh 2 & 1 \\
\vdots & \vdots & \vdots \\
-z^{(1)}(r) & \cosh r-\cosh (r-1) & 1
\end{array}\right)\left(\begin{array}{l}
a \\
b \\
c
\end{array}\right) .
$$

And then, the expression of system parameters of the new model is computed by

$$
(a, b, c)^{T}=\left(B^{T} B\right)^{-1}\left(B^{T} Y\right) .
$$

\section{Estimating the parameter $\lambda$ through Whale Optimization Algorithm}

From the previous discussion, the parameter $\lambda$ cannot be directly computed, and the parameter's function is adjusting the weight of information. Thus, the purpose of this section is to find out the values of the parameter $\lambda$.

\subsection{Building the optimization problem}

The parameter $\lambda$ not only affects the first-order generation sequence but also plays a crucial role in obtaining the best accuracy from the grey prediction model. Thus, we construct an optimization problem with constrained conditions and search for optimal value. In such a problem, the minimum MAPE is used to be the optimal model where the $\lambda$ parameter is the decision variable. The corresponding objective function is given below.

$$
\min _{\lambda} M A P E=\frac{1}{n} \sum_{k=1}^{n}\left|\frac{\hat{x}^{(0)}(k)-x^{(0)}(k)}{x^{(0)}(k)}\right| \times 100 \%,(0 \leq \lambda \leq 1),
$$




$$
\text { s.t. }\left\{\begin{array}{c}
(a, b, c)^{T}=\left(B^{T} B\right)^{-1}\left(B^{T} Y\right) \\
B=\left(\begin{array}{ccc}
-z^{(1)}(2) & \cosh 2-\cosh 1 & 1 \\
-z^{(1)}(3) & \cosh 3-\cosh 2 & 1 \\
\vdots & \vdots & \vdots \\
-z^{(1)}(n) & \cosh r-\cosh (n-1) & 1
\end{array}\right) Y=\left(\begin{array}{c}
x^{(1)}(2)-x^{(1)}(1) \\
x^{(1)}(3)-x^{(1)}(2) \\
\vdots \\
x^{(1)}(n)-x^{(1)}(n-1)
\end{array}\right) . \\
\quad+\left(x^{(0)}(1)-\frac{c}{a}\right) e^{-a(k-1)}+\frac{c}{a} \\
\hat{x}^{(1)}(k)=\frac{b}{2(a+1)}\left(e^{k}-e^{-a(k-1)+1}\right)-\frac{b}{2(a-1)}\left(e^{-k}-e^{-a(k-1)-1}\right) \\
(k)=x^{(1)}(k)-\lambda x^{(1)}(k-1)(k=2,3, \ldots, n)
\end{array}\right.
$$

\subsection{Whale Optimization Algorithm}

It's hard to calculate the parameter $\lambda$ directly from Eq. (14) as the problem is nonlinear. For the parameter $\lambda$, it requires an optimization algorithm to compute it, while making full use of the new information. This paper uses the Whale Optimization Algorithm (WOA), developed by Mirijalili and Lewis (2016), to compute the parameter $\lambda$. The WOA is an intelligent nature-inspired optimization method and is inspired by the behavior of whales hunting their prey. The WOA mainly simulates three kinds of behavior during whale hunting with high accuracy.

\section{(1) Constriction Containment Strategy}

Humpback whales can recognize prey and surround it, so the constriction containment strategy about the optimal position is for prey, and the humpback whales move towards the optimal position. The whole process can be expressed by the following equation:

$$
\begin{aligned}
& \vec{D}=|2 \vec{r} \cdot \vec{P}(t)-\vec{P}(t)| \cdot \\
& \vec{C}=2 f(t) \cdot \vec{r}-f(t) . \\
& f(i)=2-2 i / T \\
& \vec{P}(i+1)=\vec{P}^{*}-\vec{C} \cdot \vec{D}
\end{aligned}
$$

where $\vec{P}(i)$ represents the current position of the humpback whale, $\vec{P}^{*}(i)$ represents the optimal location of the population, $\vec{r}$ represents the random number on the interval $[0,1]$, and $T$ represents the maximum number of iterations.

(2) Spiral Search

Whales hunt their food in an upward spiral, and humpback whales spiral simultaneously as they surround their prey. According to the process, the data model can be constructed as follows:

$$
\vec{P}(i+1)= \begin{cases}\vec{P}^{*}(i)-(2 f(i) \cdot \vec{r}-f(i) \cdot \vec{D}), & \text { if } \xi<0.5 \\ \left|\vec{P}^{*}(i)-\vec{P}(i)\right| \cdot e^{\beta l} \cdot \cos (2 \pi l)+\vec{P}^{*}(i), & \text { if } \xi>0.5\end{cases}
$$


where $l$ represents the random number on the interval $[-1,1], \beta$ is the constant that determines the shape of the spiral motion, and $\xi$ indicates the probability of choosing enveloping contraction behavior and spiral motion.

(3) Random Walk Hunting

Humpbacks can also randomly search for food, randomly searching each other based on where the whales are. The data model can be constructed as follows:

$$
\vec{P}(i+1)=\overrightarrow{P_{r}}-\vec{C} \cdot\left|2 \vec{r} \cdot \vec{P}_{r}(i)-\vec{P}_{r}(i)\right|
$$

where $\vec{P}_{r}$ represents the location of a randomly selected individual whale in a pod $|\vec{C}|<1$, whales update their location in a random walk feeding mode.

The WOA can be used to estimate the desired parameter's value. The pseudocode of the whale optimization algorithm has been described in detail by Zhang (2019a; 2019b). In the current study, we slightly modified Zhang's code in light of our needs, and the resultant pseudocode of the WOA is presented below:

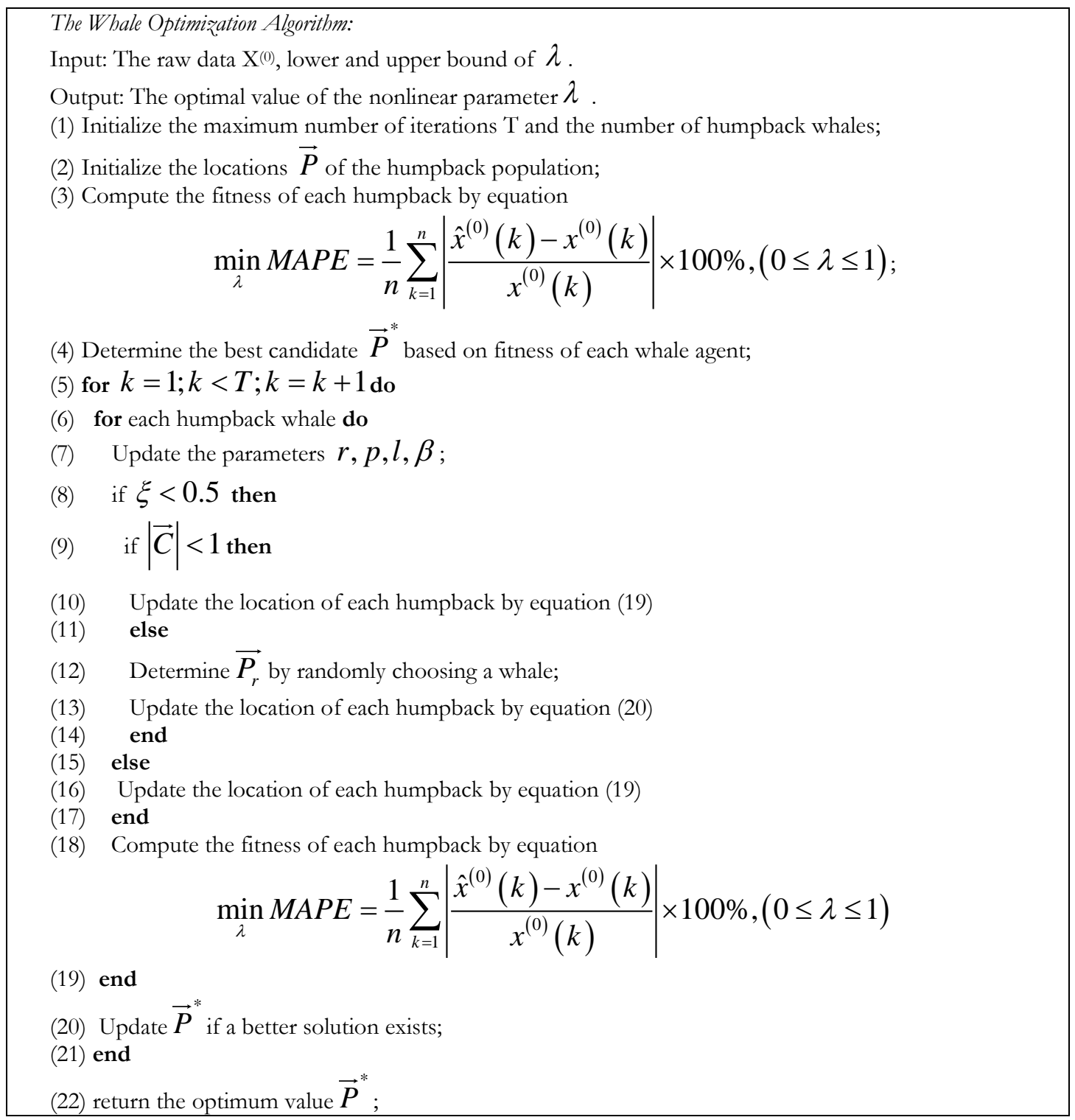


In general, the whale optimization algorithm is simple, easy to operate, and fewer parameters are regulated during algorithm operation. But the whale optimization algorithm also has some defects, such as slow convergence, late stagnation of convergence tends to fall into local optimal solution, and so on. The whale optimization algorithm is evolving.

\section{Application}

In this section, the NISinHGM $(1,1)$ is utilized to forecast the urban natural gas supply in China. By comparing to GM $(1,1)$, DGM $(1,1), \operatorname{NGM}(1,1, k)$ and NGM $(1,1, k, c)$, the feasibility of the new grey model NISinHGM $(1,1)$ is verified. The data from the year 2006 to 2019 was collected from the National Bureau of Statistics of China. In this application, the data from 2006 to 2016 are used to build the grey models, and 2017 to 2019 are used to validate the accuracy of different grey models. The raw data is shown in Table 1.

In the grey prediction model, errors are often calculated to verify the feasibility of the model. In the current study, Absolute Percentage Error (APE) and Mean Absolute Percentage Error (MAPE) are used to evaluate the accuracy of the five forecasting models. The formulas of APE and MAPE are respectively given by:

$$
\begin{gathered}
A P E(k)=\left|\frac{\hat{x}^{(0)}(k)-x^{(0)}(k)}{x^{(0)}(k)}\right| \times 100 \%,(k=2,3, \ldots) \\
M A P E=\frac{1}{n} \sum_{k=1}^{n}\left|\frac{\hat{x}^{(0)}(k)-x^{(0)}(k)}{x^{(0)}(k)}\right| \times 100 \%,(k=2,3, \ldots)
\end{gathered}
$$

Now let's see the step-by-step execution of the new model NISinHGM $(1,1)$.

Regard raw data from 2006 to 2016 will serve as the original sequence given by $X^{(0)}=(244.77,308.64,368.04,405.10,487.58,678.80,795.04,900.99,964.38,1040.79,1171.72)$.

By using the WOA algorithm, we calculated $\lambda=0.002231296$, so the first order priority accumulation sequence is computed

$\mathrm{X}^{(1)}=(244.77,309.19,368.73,405.92,488.49,679.89,796.56,902.77,966.39,1042.95,1174.05)$.

Furthermore, we make a comparison between the original sequence and first-order priority accumulation sequence, which are shown in the following Figure 1. According to the trapezoid formula, we can calculate the background sequence value

Table 1. Urban Natural Gas Supply (100 million cubic meters)

\begin{tabular}{|c|c|}
\hline Year & Raw Data \\
\hline 2006 & 244.77 \\
\hline 2007 & 308.64 \\
\hline 2008 & 368.04 \\
\hline 2009 & 405.10 \\
\hline 2010 & 487.58 \\
\hline 2011 & 678.80 \\
\hline 2012 & 795.04 \\
\hline 2013 & 900.99 \\
\hline 2014 & 964.38 \\
\hline 2015 & 1040.79 \\
\hline 2016 & 1171.72 \\
\hline 2017 & 1263.75 \\
\hline 2018 & 1443.95 \\
\hline 2019 & 1608.56 \\
\hline
\end{tabular}



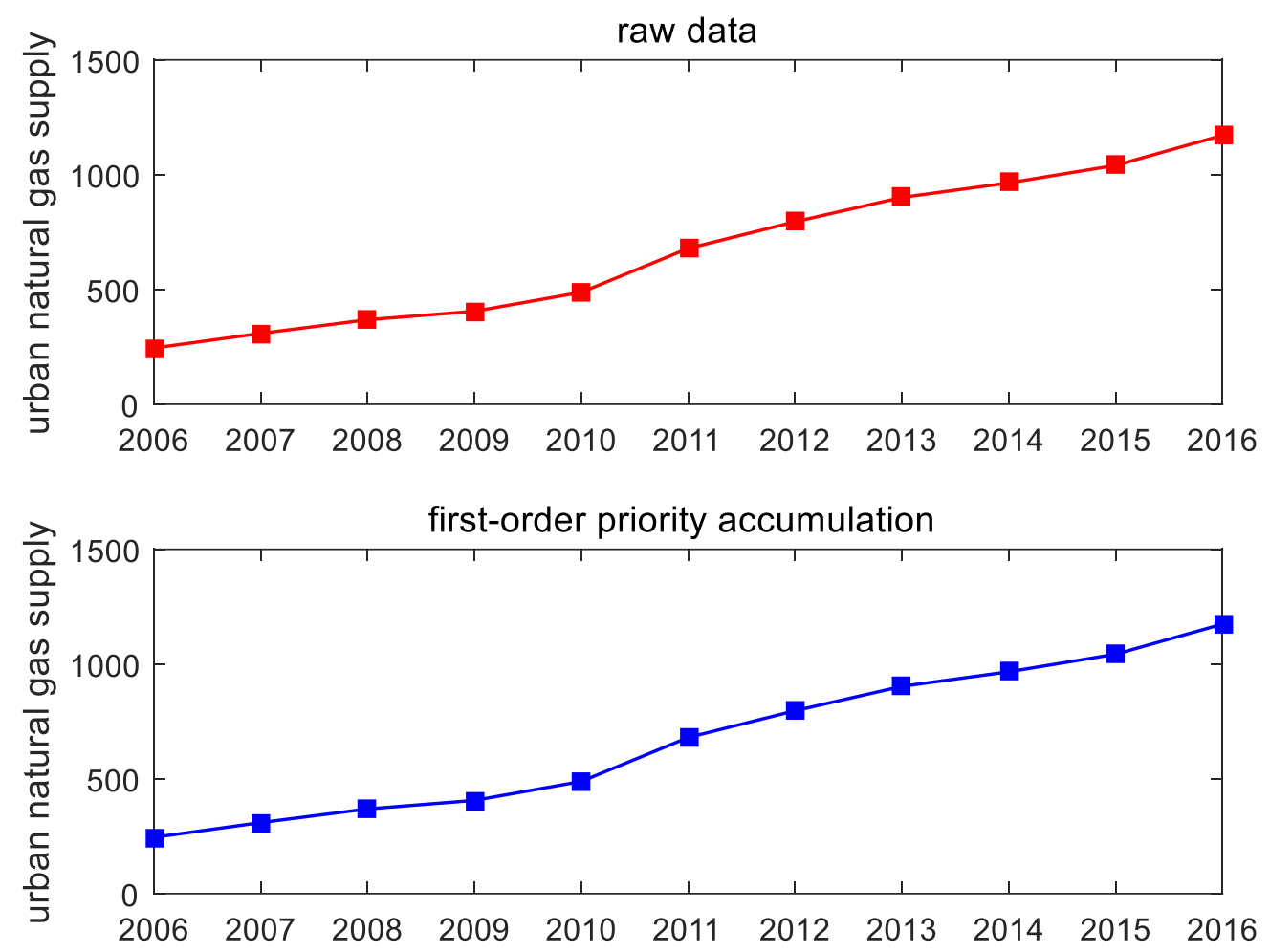

Figure 1. Comparison between raw data and first-order priority accumulation

$Z^{(1)}=(276.98,338.96,387.33,447.20,584.19,738.22,849.66,934.58,1004.67,1108.50)$.

Then, according to Theorem 2, B and $Y$ could be computed as following

$$
B=\left(\begin{array}{ccc}
-276.98 & 2.22 & 1 \\
-338.96 & 6.31 & 1 \\
-387.33 & 17.24 & 1 \\
-447.20 & 46.90 & 1 \\
-584.19 & 127.50 & 1 \\
-738.22 & 346.60 & 1 \\
-849.66 & 942.16 & 1 \\
-934.58 & 2561.06 & 1 \\
-1004.67 & 6961.69 & 1 \\
-1108.50 & 18923.84 & 1
\end{array}\right), Y=\left(\begin{array}{c}
63.87 \\
59.40 \\
37.06 \\
82.48 \\
191.22 \\
116.24 \\
105.95 \\
63.39 \\
76.41 \\
130.93
\end{array}\right) .
$$

Further, the value of the parameter $a, b, c$ can be calculated by the formula

$$
(a, b, c)^{T}=\left(B^{T} B\right)^{-1}\left(B^{T} Y\right)
$$

where

$$
\left(\begin{array}{l}
a \\
b \\
c
\end{array}\right)=\left(\begin{array}{c}
-0.0481 \\
3.2324 \\
60.5031
\end{array}\right) .
$$


Table 2. Comparison of the four model expressions

\begin{tabular}{|c|l|}
\hline GM (1,1) & $\frac{d x^{(1)}(t)}{d t}-0.1379 x^{(1)}(t)=302.0795$ \\
\hline DGM (1,1) & $x^{(1)}(k)=(1.1481)^{k-1} x^{(0)}(1)+48.05061\left[1-(1.1481)^{k-1}\right]$ \\
\hline $\mathbf{N G M}(1,1, k)$ & $\frac{d x^{(1)}(t)}{d t}+0.0214 x^{(1)}(t)=118.7708 t$ \\
\hline NGM $(1,1, k, c)$ & $\frac{d x^{(1)}(t)}{d t}--0.0182 x^{(1)}(t)=87.8866 t+86.7243$ \\
\hline
\end{tabular}

Then the expression of the NISinHGM $(1,1)$ is built

$$
\frac{d x^{(1)}(t)}{d t}-0.0481 x^{(1)}(t)=3.2324 \sinh t+60.5031
$$

In the same way, we can compute the expressions for the other models where the expressions are concluded in the following Table 2 . The entire modeling process of this application is provided in the following flowchart (see Figure 2). The results, along with the forecasting errors, obtained by the five models are shown in Tables 3, 4 and 5, and Figures 3, 4 and 5, respectively.

Later, the urban supply of natural gas is predicted by five models, which are the GM $(1,1)$ model, the DGM $(1,1)$ model, the NGM $(1,1, k)$ model, the NGM $(1,1, k, c)$ model, and the NISinHGM $(1,1)$ model. According to Figure 3, we see that the NISinHGM $(1,1)$ model predicts China's urban natural gas supply from 2006 to 2019, which most closely matches the image of the raw data. From the tables, it is clear that the forecast error of the new grey model NISinHGM $(1,1)$ is lower than the other four models. The simulation errors and forecast errors of NISinHGM $(1,1)$ only are $6.34 \%$ and $2.10 \%$, respectively. But the other four models' errors are a little high, especially the simulation error of NGM $(1,1, k)$ model is $11.32 \%$, and the forecast error of DGM $(1,1)$ model is $15.09 \%$ as high as possible. The GM $(1,1)$ forecast error is also a little high, which is $14.62 \%$. The tables and the figures confirm that the new grey model NISinHGM $(1,1)$ forecasting urban natural gas is more accurate than other grey models. Then after verifying the accuracy, we use the grey model to forecast urban natural gas supply in the next several years from 2020 to 2023, and the detailed forecast data is listed in Table 5 .

According to the prediction data for 2020 to 2023, the urban natural gas supply is increasing year by year. As we know, natural gas produces less pollution and is economical energy; thus, an increase in its demand is very likely. Also, the natural gas is delivered to some distant mountains, showing its outreach and exceeding demand in the population as remote as possible. Thus, the prediction of increasing urban natural gas supply is logical. It is argued that the increase in the urban natural gas supply is due to a significant increase in natural gas consumption in the power generation, residential, transportation, and chemical industries. To meet the demand for natural gas in various fields, the governments and policy-decision makers should try to produce natural gas, and increase the natural gas supply to the city as much as possible.

\section{Conclusion}

The study proposes a new grey forecasting model NISinHGM $(1,1)$ to forecast the urban natural gas supply. A new information priority accumulation method has been integrated into the grey model with hyperbolic sine driving term. Firstly, we use the Whale Optimization Algorithm to search for an important parameter generated in the new information priority accumulation method. Then we make use of grey prediction theory and ordinary differential equation theory to determine the model. In the end, we verify the validity of the proposed grey model by forecasting urban natural gas supply. Comparative analysis with four models revealed the superiority of the proposed model, which revealed better forecast accuracy. 


\section{Application in urban natural gas supply}

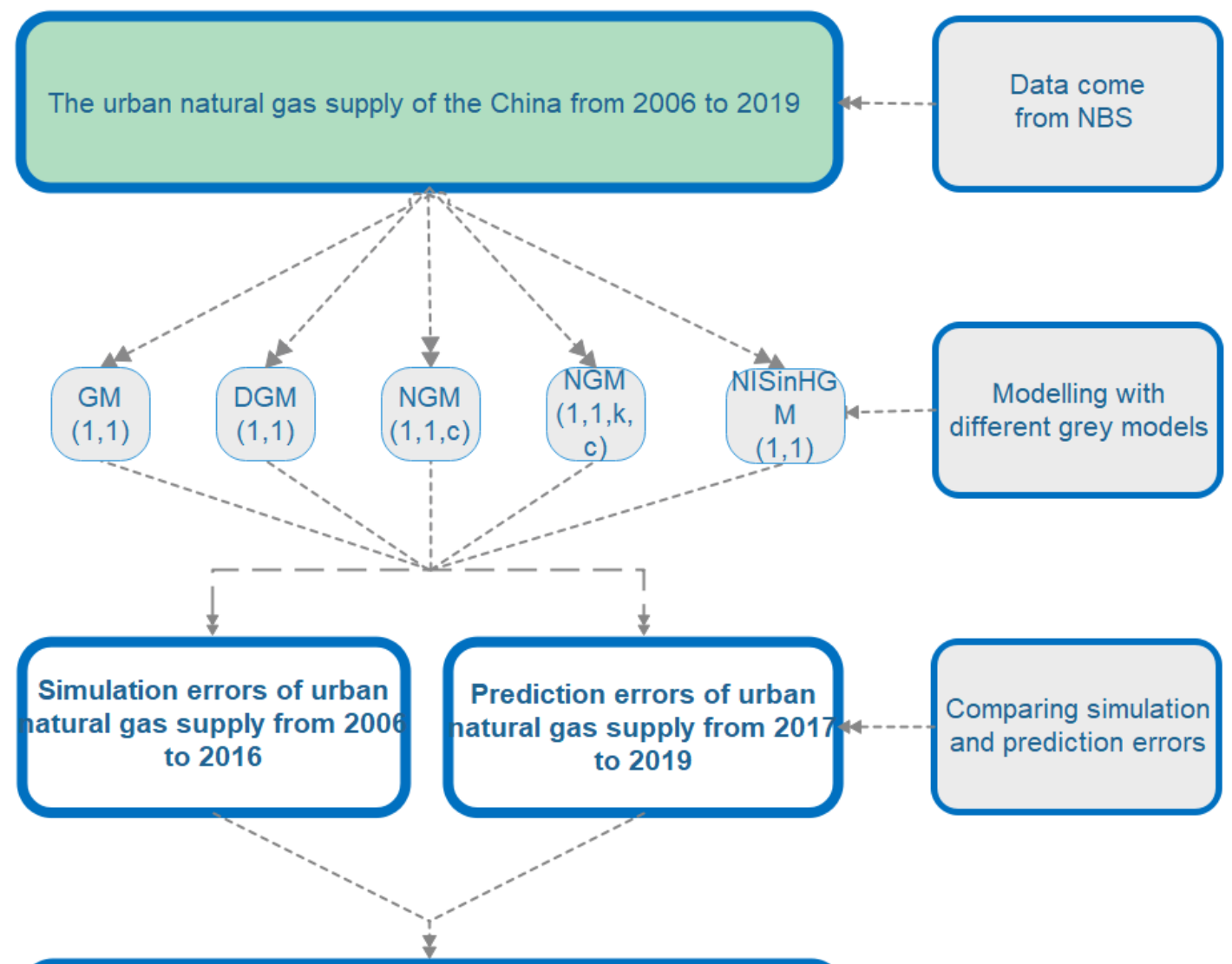

Forecasting short-term urban natural gas supply from 2020 to 2023 by grey models

Figure 2. The flowchart of the modeling process

Table 3. The calculation results of urban natural gas supply

\begin{tabular}{|c|c|c|c|c|c|c|}
\hline Year & Raw data & GM (1,1) & DGM (1,1) & NGM (1,1,k) & NGM (1,1, $\boldsymbol{k}, \boldsymbol{c})$ & NISinHGM (1,1) \\
\hline 2006 & 244.77 & 244.77 & 244.77 & 244.77 & 244.77 & 244.77 \\
\hline 2007 & 308.64 & 360.08 & 360.69 & 171.30 & 224.92 & 318.27 \\
\hline 2008 & 368.04 & 413.32 & 414.11 & 285.20 & 317.74 & 395.80 \\
\hline 2009 & 405.10 & 474.42 & 475.43 & 396.68 & 412.26 & 477.16 \\
\hline 2010 & 487.58 & 544.56 & 545.84 & 505.81 & 508.53 & 562.53 \\
\hline 2011 & 678.80 & 625.06 & 626.67 & 612.63 & 606.56 & 652.10 \\
\hline 2012 & 795.04 & 717.47 & 719.48 & 717.20 & 706.39 & 746.10 \\
\hline 2013 & 900.99 & 823.54 & 826.02 & 819.56 & 808.05 & 844.75 \\
\hline 2014 & 964.38 & 945.29 & 948.35 & 919.75 & 911.59 & 948.32 \\
\hline 2015 & 1040.79 & 1085.04 & 1088.79 & 1017.83 & 1017.02 & 1057.14 \\
\hline 2016 & 1171.72 & 1245.44 & 1250.02 & 1113.83 & 1124.39 & 1171.72 \\
\hline 2017 & 1263.75 & 1429.57 & 1435.14 & 1207.80 & 1233.73 & 1293.02 \\
\hline 2018 & 1443.95 & 1640.91 & 1647.67 & 1299.79 & 1345.08 & 1423.21 \\
\hline 2019 & 1608.56 & 1883.50 & 1891.67 & 1389.84 & 1458.47 & 1567.73 \\
\hline
\end{tabular}


Table 4. Calculation errors under different models

\begin{tabular}{|c|c|c|c|c|c|}
\hline Year & GM (1,1) & DGM (1,1) & NGM (1,1,k) & NGM (1,1, $\boldsymbol{k}, \boldsymbol{c})$ & NISinHGM (1,1) \\
\hline 2006 & 0.00 & 0.00 & 0.00 & 0.00 & 0.00 \\
\hline 2007 & 16.67 & 16.87 & 44.50 & 27.13 & 3.12 \\
\hline 2008 & 12.30 & 12.52 & 22.51 & 13.67 & 7.54 \\
\hline 2009 & 17.11 & 17.36 & 2.08 & 1.77 & 17.79 \\
\hline 2010 & 11.69 & 11.95 & 3.74 & 4.30 & 15.37 \\
\hline 2011 & 7.92 & 7.68 & 9.75 & 10.64 & 3.93 \\
\hline 2012 & 9.76 & 9.50 & 9.79 & 11.15 & 6.16 \\
\hline 2013 & 8.60 & 8.32 & 9.04 & 10.31 & 6.24 \\
\hline 2014 & 1.98 & 1.66 & 4.63 & 5.47 & 1.66 \\
\hline 2015 & 4.25 & 4.61 & 2.21 & 2.28 & 1.57 \\
\hline 2016 & 6.29 & 6.68 & 4.94 & 4.04 & 0.00 \\
\hline 2017 & 13.12 & 13.56 & 4.43 & 2.38 & 2.32 \\
\hline 2018 & 13.64 & 14.11 & 9.98 & 6.85 & 1.44 \\
\hline 2019 & 17.09 & 17.60 & 13.60 & 9.33 & 2.54 \\
\hline MAPEsimu & 9.66 & 9.72 & 11.32 & 9.08 & 6.34 \\
\hline MAPEfore & 14.62 & 15.09 & 9.34 & 6.18 & 2.10 \\
\hline MAPEtotal & 10.80 & 10.96 & 10.86 & 8.41 & 5.36 \\
\hline
\end{tabular}

Table 5. Comparison of five model predictions

\begin{tabular}{|c|c|c|c|c|c|}
\hline Year & GM (1,1) & DGM (1,1) & NGM (1,1,k) & NGM (1,1, $\boldsymbol{k}, \boldsymbol{c})$ & NISinHGM (1,1) \\
\hline 2020 & 2161.95 & 2171.80 & 1477.98 & 1573.95 & 1740.90 \\
\hline 2021 & 2481.56 & 2493.42 & 1564.26 & 1691.55 & 1981.12 \\
\hline 2022 & 2848.43 & 2862.67 & 1648.72 & 1811.31 & 2392.22 \\
\hline 2023 & 3269.54 & 3286.60 & 1731.39 & 1933.26 & 3255.91 \\
\hline
\end{tabular}

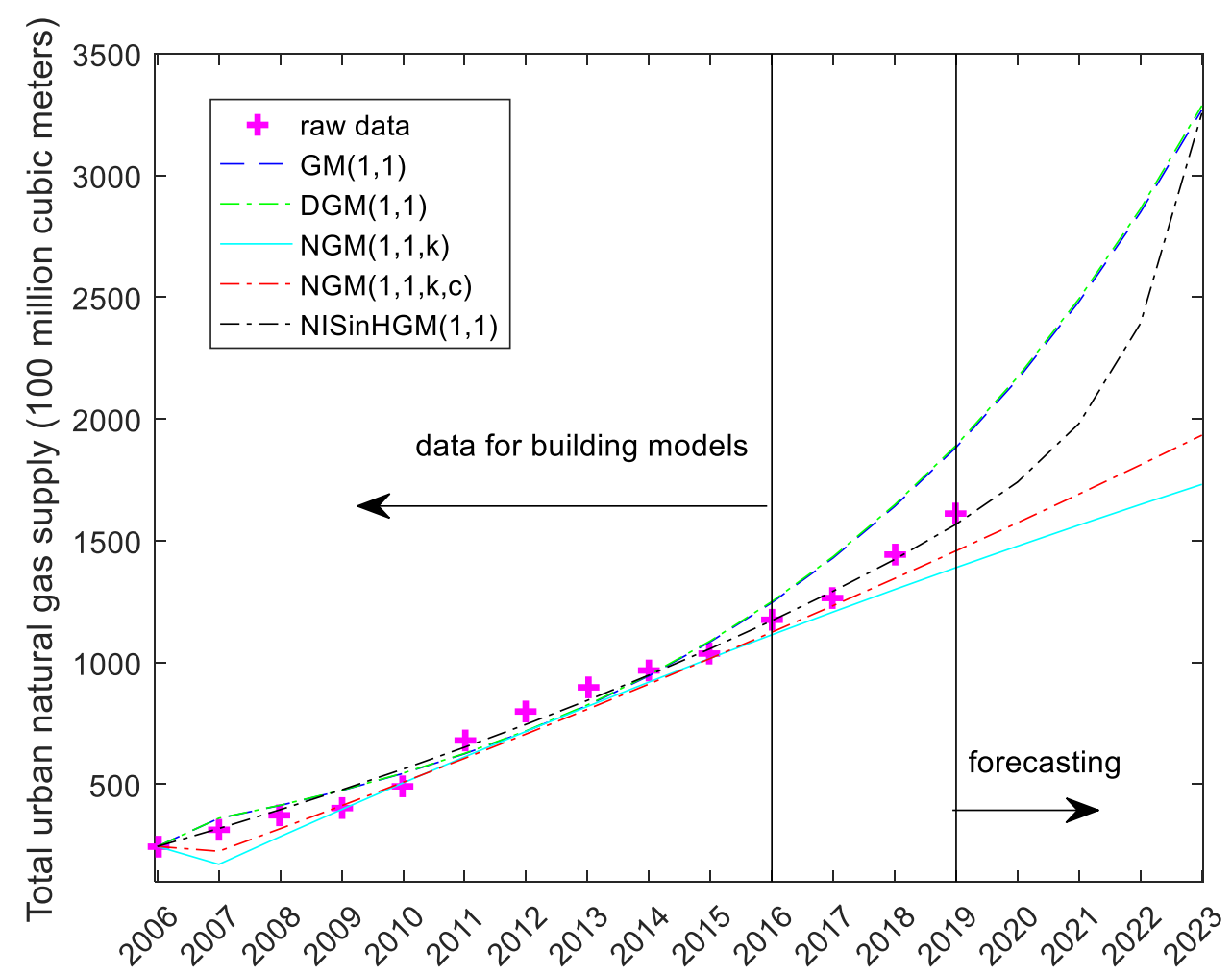

Figure 3. Five models' calculation results of urban natural gas supply 


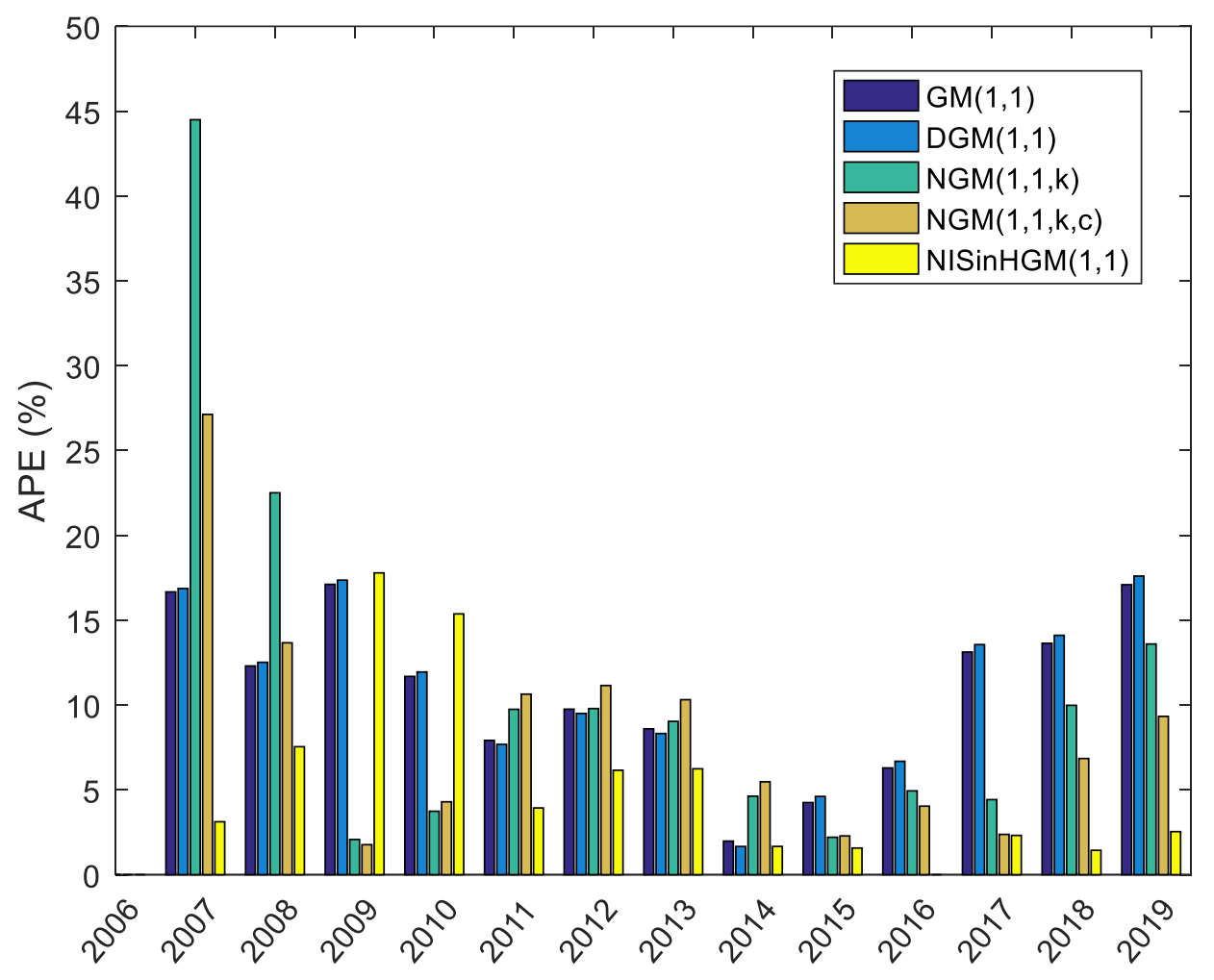

Figure 4. Absolute Percentage Error graphs under five models

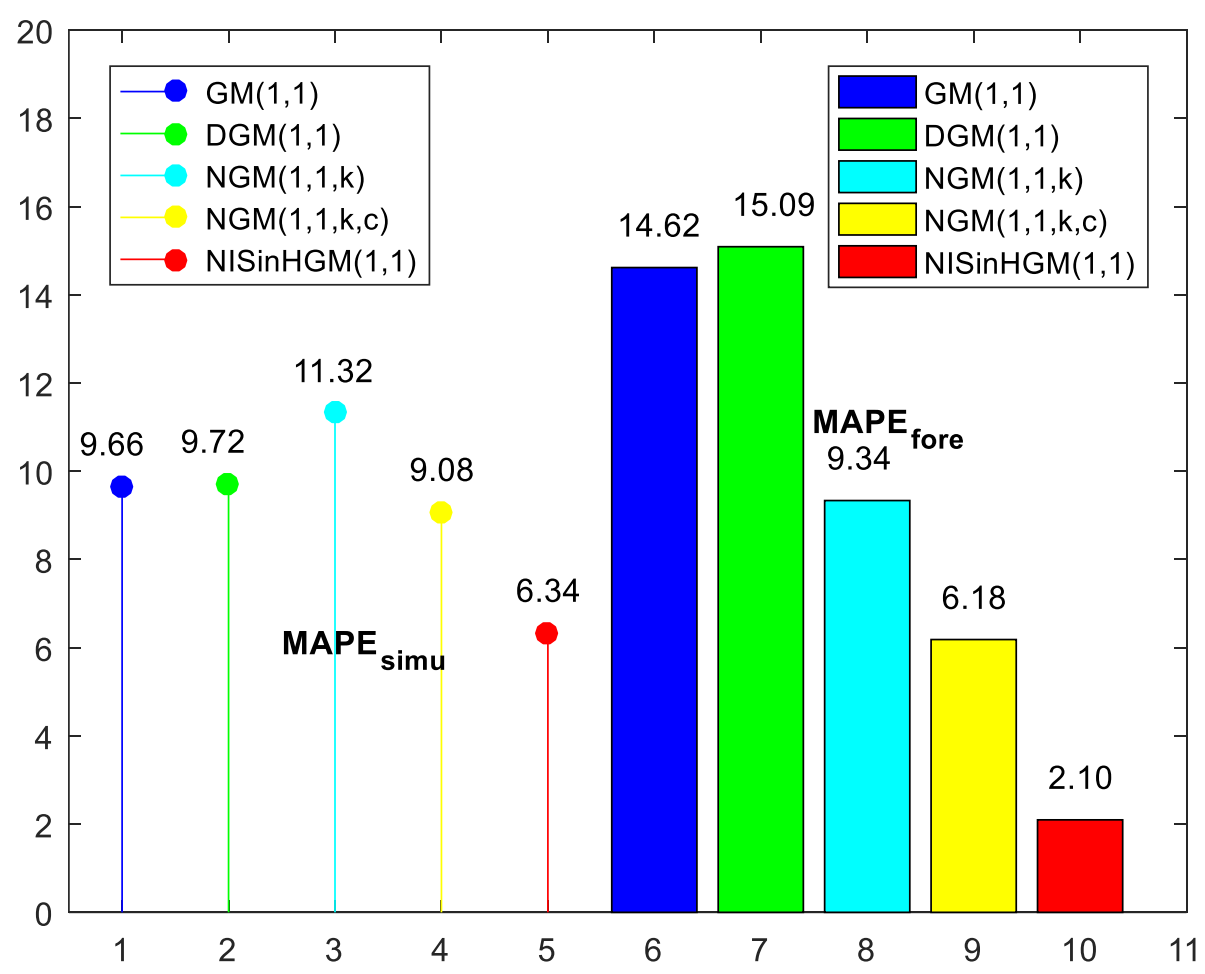

Figure 5. Comparison of Mean Absolute Percentage Errors of five forecasting models 
The current study demonstrates that moderate improvement in the grey model is possible with the aid of the new information priority methods, and the resultant model can outperform the original model in terms of accuracy. Combining the new information priority accumulation method into the grey model is a good way to improve the accuracy and increase its range of applicability. In the future, some other multivariate forecasting models with new information priority accumulation will be considered.

\section{Acknowledgement}

This research was supported by the National Natural Science Foundation of China (No. 72001181, 71901184, 72071023, 71771033), the Humanities and Social Science Project of Ministry of Education of China (No. 19YJCZH119), the National Statistical Scientific Research Project (No.2018LY42), the funding of V.C. \& V.R. Key Lab of Sichuan Province (SCVCVR2020.01VS), the Key R \& D projects of Sichuan Science and Technology Department (No.2021YFG0031), the funding of Research center of Sichuan County Economy, Development (No. xy2020042), and the Open Fund (PLN 201710) of State Key Laboratory of Oil and Gas Reservoir Geology and Exploitation (Southwest Petroleum University), and the fund of Research on the model of characteristic factors and development trend of private economy in Sichuan province based on big data.

\section{References}

Chen, P.-Y., \& Yu, H.-M. (2014). Foundation settlement prediction based on a novel NGM Model. Mathematical Problems in Engineering, 2014, Article ID 242809. https://doi.org/10.1155/2014/242809

Cui, J., Liu, S., Zeng, B., \& Xie, N. (2013). A novel grey forecasting model and its optimization. Applied Mathematical Modelling, 37(6), 4399-4406. https://doi.org/10.1016/j.apm.2012.09.052

Deng, C.-Y., Zhang, S.-T., \& Deng, J.-L. (2012). Numerical Mapping in DNA Sequences and Analysis of the Genetic Information by GM(1,N). The Journal of Grey System, 24(3), 217-224.

Deng, J. (1982). Control problems of grey systems. Systems \& Control Letters, 1(5), $288-294$. https://doi.org/10.1016/S0167-6911(82)80025-X

Ma, X. Liu, Z., \& Chen, Y. (2014). Optimizing the Grey GM(1,N) Model by Rebuilding All the Back Ground Values. Journal of Systems Science \& Information, 2(6):543-552. http://syssci.ac.cn/EN/abstract/abstract55.shtml

Ma, X., Wu, W., Zeng, B., Wang, Y., \& Wu., X. (2019). The conformable fractional grey system model. IS A Transactions, 96, 255-271. https://doi.org/10.1016/j.isatra.2019.07.009.

Mirjalili, S., \& Lewis, A. (2016). The Whale Optimization Algorithm. Advances in Engineering Software, 95, 5167. https://doi.org/10.1016/J.ADVENGSOFT.2016.01.008

Tien, T.-L. (2005). The indirect measurement of tensile strength of material by the grey prediction model GMC(1, n). Measurement Science and Technology, 16(6), 1322-1328. https://doi.org/10.1088/09570233/16/6/013

Wu, L., \& Zhang, Z. (2018). Grey multivariable convolution model with new information priority accumulation. Applied Mathematical Modelling, 62, 595-604. https://doi.org/10.1016/J.APM.2018.06.025

Wu, L., Liu, S., Yao, L., Yan, S., \& Liu, D. (2013). Grey system model with the fractional order accumulation. Communications in Nonlinear Science and Numerical Simulation, 18(7), 1775-1785.

Wu, W., Ma, X., Wang, Y., \& Zeng, B. (2019b). Research on a novel fractional GM( $\alpha$, n) model and its applications. Grey Systems: Theory and Application, 9(3), 356-373. https://doi.org/10.1108/GS-11-20180052

Wu, W., Ma, X., Zeng, B., Wang, Y., \& Cai, W. (2018). Application of the novel fractional grey model FAGMO $(1,1, \mathrm{k})$ to predict China's nuclear energy consumption. Energy, 165, 223-234. https://doi.org/10.1016/j.energy.2018.09.155

Wu, W., Ma, X., Zeng, B., Wang, Y., \& Cai, W. (2019). Forecasting short-term renewable energy consumption of China using a novel fractional nonlinear grey Bernoulli model. Renewable Energy, 140, 70-87. https://doi.org/10.1016/j.renene.2019.03.006

Xia, J., Ma, X., Wu, W., Huang, B., \& Li, W. (2020). Application of a new information priority accumulated grey model with time power to predict short-term wind turbine capacity. Journal of Cleaner Production, 244, 118573. https://doi.org/10.1016/j.jclepro.2019.118573 
Xie, N.-M., Liu, S.-F., Yang, Y.-J., \& Yuan, C.-Q. (2013). On novel grey forecasting model based on nonhomogeneous index sequence. Applied Mathematical Modelling, 37(7), 5059-5068. https://doi.org/10.1016/j.apm.2012.10.037

Zhang, P., Ma, X., \& She, K. (2019a). A Novel Power-Driven Grey Model with Whale Optimization Algorithm and Its Application in Forecasting the Residential Energy Consumption in China. Complexity, 2019, Article ID 1510257. https://doi.org/10.1155/2019/1510257

Zhang, P., Ma, X., \& She, K. (2019b). Forecasting Japan's Solar Energy Consumption Using a Novel Incomplete Gamma Grey Model. Sustainability, 11(21), 5921. https://doi.org/10.3390/SU11215921

Zhou, W.-J., Zhang, H.-R., Dang, Y.-G., \& Wang, Z.-X. (2017). New information priority accumulated grey discrete model and its application. Chinese Journal of Management Science, 25(8),140-148. https://doi.org/10.16381/j.cnki.issn1003-207x.2017.08.015 